\title{
Streamlining the management of Parkinson disease in Canada
}

\author{
Veronica Bruno MD MPH, Maria Eliza Thomaz de Freitas MD
}

- Cite as: CMAJ 2019 September 9;191:E979-80. doi: 10.1503/cmaj.191089

See related article at www.cmaj.ca/lookup/doi/10.1503/cmaj.181504

A $\mathrm{n}$ updated Canadian guideline on the management of Parkinson disease, produced by a collaboration of specialists, with sponsorship from Parkinson Canada, represents a great effort to streamline the management of Parkinson disease across Canada. ${ }^{1}$ Since the publication of the first Canadian guideline on Parkinson disease in 2012, ${ }^{2}$ substantial progress has been made both in Canada and internationally in research and understanding of optimal management of the disease. After a comprehensive analysis of international guidelines, ${ }^{3-6}$ as well as of meta-analyses, systematic reviews and clinical trials published between 2006 and 2016, the guideline panel developed a set of practical recommendations that are applicable and relevant to the Canadian health system.

The guideline provides evidence-based recommendations to improve the overall standard of care of individuals with Parkinson disease in Canada, not only for health care professionals, but also for policy-makers, patients themselves and their caregivers. Managing the complexity of Parkinson disease requires clear, standardized procedures that can be used by all actors involved. We discuss the new guideline in the current international context, highlighting what is novel, and discuss questions that must still be addressed by research.

Parkinson disease is among the most prevalent chronic neurodegenerative conditions. ${ }^{7}$ The combination of motor and nonmotor symptoms, variable response to treatments and the potential adverse effects of medications pose multiple challenges to those with the condition, their caregivers and their health care providers.

In Canada, according to the National Population Health Study of Neurological Conditions, the prevalence of Parkinson disease was 170 per 100000 people in 2013/14. ${ }^{2}$ With a growing and aging population, the number of Canadians older than 40 years who are living with Parkinson disease will increase by $65 \%$ by 2031 , and the number of Canadians older than 65 years living with Parkinson disease will more than double. ${ }^{8}$

We highlight 6 of the most important aspects of the updated Canadian guideline. First, the guideline underscores the importance of empowering patients and improving communication, with an emphasis on shared decision-making. The recommendations

\section{KEY POINTS}

- An updated Canadian guideline for Parkinson disease represents a great effort to streamline the management of Parkinson disease across Canada.

- Since the 2012 version, progress has been made in the management of advanced Parkinson disease, including the use of deep brain stimulation, intrajejunal levodopa infusion and the recently approved use of apomorphine.

- Further developments in the understanding and management of Parkinson disease are still needed, including treatments with improved adverse effect profiles and therapeutics for the broad spectrum of nonmotor symptoms of Parkinson disease.

are complete, concise and reader friendly for different audiences. Each recommendation is based on the best current evidence, written in medical language and accompanied by an explanatory paragraph in layperson's language.

Second, the guideline suggests the use of the most recent (2015) International Parkinson and Movement Disorder Society criteria for the diagnosis of Parkinson disease. ${ }^{9}$ These criteria help physicians to systematize the process of diagnosis and help to ensure reproducibility across centres. They are applicable by clinicians with more limited expertise in recognizing the disease.

Third, in a substantial change from 2012, the guideline emphasizes that advanced therapies such as deep brain stimulation and intrajejunal levodopa-carbidopa gel infusion are now routinely used in Parkinson disease to manage motor symptoms and fluctuations. Since the previous guideline, there have been important advances in the management of motor and nonmotor symptoms of Parkinson disease. Deep brain stimulation of the subthalamic nucleus or globus pallidus interna is effective (grade of recommendation: A) against motor complications and levodopa-induced dyskinesia and is currently the surgical treatment of choice in selected patients. Intrajejunal levodopacarbidopa gel infusion can be considered for patients with debilitating drug complications. Both procedures are now available in many centres in Canada and are routinely used in patients with Parkinson disease. 
Fourth, the guideline reflects Health Canada's recent approval of subcutaneous apomorphine for the acute intermittent treatment of "off" episodes - the periods when levodopa stops working optimally and symptoms recur. It is important to note that apomorphine should be used only as adjunctive therapy in patients who are receiving optimized treatment for Parkinson disease and still have motor fluctuations, and always under the care of a physician with experience in the diagnosis and management of the disease.

Fifth, a warning that impulse control disorders can develop in a person with Parkinson disease who is on any dopaminergic therapy, at any stage in the disease course, is of capital relevance for health care providers caring for patients who are being treated for Parkinson disease. Impulse control disorders are repetitive reward-based behaviours such as pathological gambling, hypersexuality, binge eating and compulsive shopping. Their main feature is the inability of a patient to resist the impulse to perform an action that can harm the patient or others. ${ }^{10}$ Recent studies have shown that the 5 -year cumulative incidence of impulse control disorders in patients with Parkinson disease taking dopamine agonists is about $46 \%{ }^{11}$

Lastly, an important new inclusion in the updated guideline is the recommendation that palliative care be offered to patients with Parkinson disease, during all stages of the disease - not only for end-of-life decisions, but also for discussions about prognosis, advanced care planning and recommendations on available support services such as home safety and long-term care facilities, among others. Furthermore, the option of medical assistance in dying should be considered for discussion if the patient asks for information about it.

This updated Canadian guideline on the management of Parkinson disease provides a comprehensive review of the disease with an emphasis on communication, diagnosis and progression, treatment and nonmotor features, and the new addition of recommendations of palliative care, but the authors recognize that there are important gaps in knowledge. More research is needed to increase the current understanding of Parkinson disease biomarkers for neuroprotective and disease-modifying treatments, and improved therapies for motor and nonmotor symptoms. Until neuroprotective and disease-modifying treatments become available, improvements in the management of motor fluctuations and dyskinesia, such as long-lasting treatments with a reduced adverse effect profile, are also needed. Evaluation of promising nonpharmacologic treatments must also be systematic, to allow for their inclusion in evidence-based recommendations.

Finally, although there has been improvement in recognizing the relevance of nonmotor symptoms of Parkinson disease, adequate treatment options are still lacking for problems such as anxiety, sleep disturbances, constipation, overactive bladder symptoms, pain, fatigue and apathy. Potential treatments for those disabling symptoms must be explored, as they can have a substantial negative impact on patients' quality of life.

\section{References}

1. Grimes D, Fitzpatrick M, Gordon J. Canadian guideline for Parkinson disease. CMAJ 2019;191:E989-1004.

2. Grimes D1, Gordon J, Snelgrove B, et al.; Canadian Neurological Sciences Federation. Canadian guidelines on Parkinson's disease. Can J Neurol Sci 2012; 39(Suppl 4):S1-30.

3. Parkinson's disease in adults: diagnosis and management. London (UK): National Institute for Health and Care Excellence (UK); 2017.

4. Diagnosis and pharmacological management of Parkinson's disease: a national clinical guideline. Edinburgh: Scottish Intercollegiate Guidelines Network; 2010.

5. Fox SH, Katzenschlager R, Lim SY, et al. The movement disorder society evidencebased medicine review update: treatments for the motor symptoms of Parkinson's disease. Mov Disord 2011;26 Suppl 3:S2-41

6. Ferreira JJ, Katzenschlager R, Bloem BR, et al. Summary of the recommendations of the EFNS/MDS-ES review on therapeutic management of Parkinson's disease. Eur J Neurol 2013;20:5-15.

7. Pringsheim T, Jette N, Frolkis A, et al. The prevalence of Parkinson's disease: a systematic review. Mov Disord 2014;29:1583-90.

8. Gaskin J, Gomes J, Darshan S, et al. Burden of neurological conditions in Canada. Neurotoxicology 2017;61:2-10.

9. Postuma RB, Berg D, Stern M, et al. MDS clinical diagnostic criteria for Parkinson's disease. Mov Disord 2015;30:1591-601.

10. Weintraub D, David AS, Evans AH, et al. Clinical spectrum of impulse control disorders in Parkinson's disease. Mov Disord 2015;30:121-7.

11. Corvol J-C, Artaud F, Cormier-Dequaire F, et al. Longitudinal analysis of impulse control disorders in Parkinson disease. Neurology 2018;91:e189-201.

\section{Competing interests: None declared.}

This article was solicited and has not been peer reviewed.

Affiliations: Department of Clinical Neurosciences, Movement Disorders Program (Bruno) and Hotchkiss Brain Institute (Bruno), University of Calgary, Calgary, Alta.; Division of Neurology, Department of Medicine (Thomaz de Freitas), McMaster University, Hamilton, Ont.
Contributors: Veronica Bruno conceptualized and designed the work, and wrote the original draft. Maria Eliza Thomaz de Freitas contributed to the conception of the work and writing of the manuscript. Both authors contributed to revising the manuscript critically for important intellectual content, gave final approval of the version to be published and agreed to be accountable for all aspects of the work.

Correspondence to: Veronica Bruno, veronica.bruno@ucalgary.ca 